\title{
How Smart LEDs Lighting Benefit Color Temperature and Luminosity Transformation
}

\author{
Yu-Sheng Huang ${ }^{1}$, Wei-Cheng Luo ${ }^{1}$, Hsiang-Chen Wang ${ }^{1}$, Shih-Wei Feng ${ }^{2}$, Chie-Tong Kuo ${ }^{3}$ \\ and Chia-Mei $\mathrm{Lu}^{4, *}$ \\ 1 Graduate Institute of Opto-Mechatronics, National Chung Cheng University, 168 University Rd., \\ Min-Hsiung, Chia-Yi 62102, Taiwan; yushenghuang418@gmail.com (Y.-S.H.); chch833@gmail.com (W.-C.L.); \\ hcwang95@gmail.com (H.-C.W.) \\ 2 Department of Applied Physics, National University of Kaohsiung, 700 Kaohsiung University Rd., \\ Nanzih District, Kaohsiung 81148, Taiwan; swfeng@nuk.edu.tw \\ 3 Department of Physics, National Sun Yat-sen University, 70 Lienhai Rd., Kaohsiung 80424, Taiwan; \\ ctkuo@mail.nsysu.edu.tw \\ 4 Department of Digital Multimedia Design, Cheng Shiu University, 840 Chengcing Rd., Niaosong District, \\ Kaohsiung 83347, Taiwan \\ * Correspondence: K1024@gcloud.csu.edu.tw; Tel.: +886-7-735-8800 (ext. 5508) \\ Academic Editor: Jean-Michel Nunzi
}

Received: 20 February 2017; Accepted: 5 April 2017; Published: 11 April 2017

\begin{abstract}
Luminosity and correlated color temperature (CCT) have gradually become two of the most important factors in the evaluation of the performance of light sources. However, although most color performance evaluation metrics are highly correlated with $\mathrm{CCT}$, these metrics often do not account for light sources with different CCTs. This paper proposes the existence of a relationship between luminosity and CCT to remove the effects of CCT and to allow for a fairer judgment of light sources under the current color performance evaluation metrics. This paper utilizes the Hyper-Spectral Imaging (HSI) technique to recreate images of a standard color checker under different luminosities, $\mathrm{CCT}$, and light sources. The images are then analyzed and transformed into interpolation figures and equal color difference curves. This paper utilizes statistic tools and symmetry properties to determine an exponential relationship between luminosity and CCT in red-green-blue (RGB) LED and OLED light sources. Such a relationship presents an option to remove the effects of CCT in color evaluation standards, as well as provide a guide line for adjusting visual experience solely by adjusting luminosity when creating a lighting system.
\end{abstract}

Keywords: correlated color temperature (CCT); hyper-Spectral Imaging (HSI); color performance evaluation

\section{Introduction}

In recent decades, the higher emitting rate and low pollution characteristics of LED lights have gradually led to their replacing regular incandescent bulbs and becoming the new generation light source [1-7]. However, various color evaluation methods have proven to be inconsistent or even inappropriate in previous studies. The color rendering index (CRI) standard, which is the gold standard for evaluating the performance of light sources, is inconsistent for use in evaluating LED light sources because of its emission characteristic [8,9]. Newly proposed color evaluation metrics such as color quality scale (CQS) [10], feeling of contrast index (FCI), color discrimination index (CDI), and color preference index (CPI) also failed to measure LED light sources fairly [11-17]. Moreover, these color evaluation standards are often measured under the same CCT, which are not appropriate for measuring LED light sources performance [18-24]. 
Luminosity and correlated color temperature (CCT) have become the two of the most important factors in the evaluation of the performance of light sources $[18,21,25]$. Kruithof introduced the "Kruithof curve" to describe the change in luminosity and CCT's effect on visual experience in generating pleasing feelings on images [26]. Both Fotios and McNelis indicated that under the same CRI, the light sources with higher CCT have higher brightness and visual experience and are more preferred $[27,28]$. Ju pointed out that under the same luminosity, higher CCT light sources have higher brightness visual experiences [29]. Houser and his colleagues utilized statistical tools and confirmed the high correlation between CCT and various common color evaluation standards such as CRI and CQS. CCT should not be used as a color evaluation standard because it is often predetermined before the creation of any light and lighting system; thus Huser further suggested the removal of CCT from color evaluation standards to provide more accurate color performance evaluation metrics [30-33].

The purpose of these color evaluation standards is to quantize the visual experiences observed by human eyes. Although visual experiences are subjective, the visual experiences observed can be simulated by primary color receptors in human eyes. This visual experience originates from the receiving light reflected from reflective surfaces. Hence, the reflected light can be considered as an integration of light source spectrum and surface reflective spectra [11]. The stimulus of receptors in human eyes can also be defined as the integration of corresponding color spectra and its characteristic function. While the surface reflective spectrum and the receptors' characteristic function can be difficult to obtain, the light source spectrum can be replaced to stimulate the corresponding visual experience. This study utilized hyper-spectral imaging and color reproduction technique to obtain new images under different luminosity and light source setup. This novel approach of lighting image reproduction, here referred to as "smart" lighting reproduction, allowed fast and decently accurate analysis on lighting performance.

This study focused on the influence of CCT. Despite the considerable effect of CCT on color performance, most color evaluation does not take this effect into account. Most studies have highlighted the significant relationship between CCT and brightness experience, and luminosity is often considered in most standard color evaluation metrics $[29,34,35]$. Thus, the intention of this study was to create a transformation relationship between CCT and luminosity. This study attempted to match metamerism under different light sources and compare to corresponding under different light sources. The same visual experience can be reached by adjusting CCT and luminosity through this relationship and reasonable separation of CCT from the current color performance evaluation standards can be achieved.

\section{Methods}

A photo of the classic Macbeth color checker was taken under CIE standard light source D65 under 400 lux via digital camera $30 \mathrm{~cm}$ above. This photo was processed using Hyper-Spectral Imaging (HSI) combined with color reproduction technique that simulated identical images under different correlated color temperature (CCT) and luminosity with CIE, CIEF, RGB LED, and OLED standard light sources. Information related to the HSI technique will be discussed in Section 2.2. These images were compared with the original image and the average color difference was calculated under CIE DE2000 formula. This average color difference was then processed and analyzed. A summary of the full process is shown in Figure 1. 


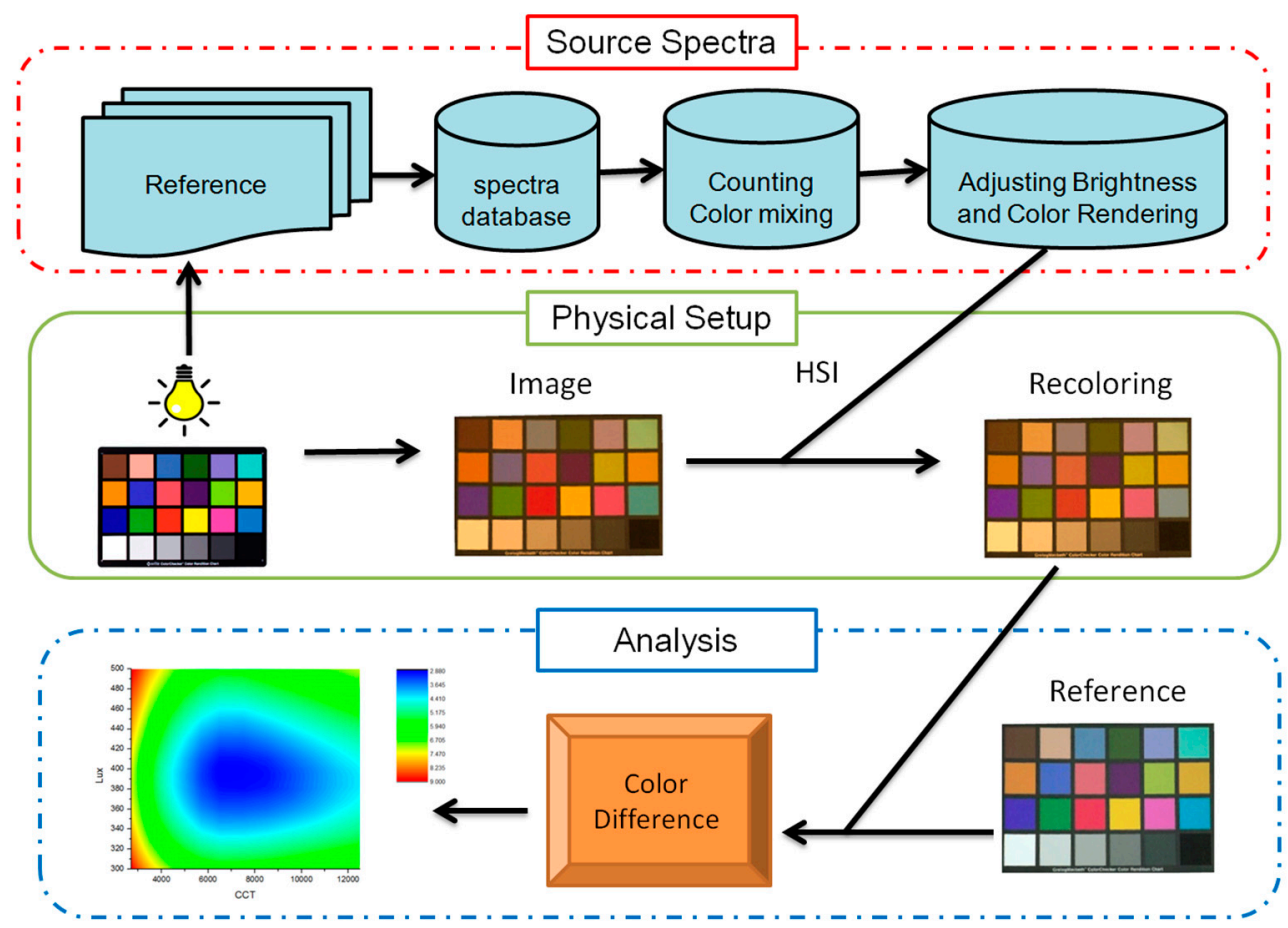

Figure 1. Flow chart of the procedure used in this paper to analyze the relationship between luminosity and correlated color temperature (CCT). The whole process can be separate into three large categories: Hyper-Spectral Imaging (HSI), image processing, and analysis.

\subsection{Reference Image Setup}

The goal of this study was to understand how CCT and luminosity affect human perception of a certain image. This study sought to determine the relationship between CCT and luminosity while maintaining the same visual effect for human eyes. The CIE standard light source D65 under luminosity 400 lux was used as an imaging reference point. The image was taken $30 \mathrm{~cm}$ above the color checker. This reference point had the advantage of being similar to normal office working light source and height setup, Moreover, the reference point had a relatively small color difference with the simulated images, thereby increasing the accuracy of the analysis. However, some comparisons with the image reference point result in the lowest color difference remain above 0 . In this case, the points with the smallest color difference as center points are referred to. This study also used different light sources, CCT, and luminosities as imaging reference points. These attempts are discussed in later sections.

\subsection{Hyper-Spectral Imaging and Color Reproduction}

Hyper-Spectral Imaging is a technique that acquires the spectral imaging of an object. Combined with color reproduction via programming, it is possible to reproduces new images by replacing the light source spectrum. Due to the complication of the process of imaging reproduction, only a brief summary of such a technique is provided. More details can be find in references [11,36-43]. Image reproduction process can be separated into three parts: hyper-spectral analysis, finding spectrum replacing matrix, and replacing image through the switching spectrum. When light interacts with an object's surface, an image is created after the reflection. This image can be written as an integral part of source and reflecting spectra (which is related to the surface). The image reproduction technique developed in this study allows the user to replace the source spectrum with known standard light source spectrum. Figure 2 shows the light spectra used in the study. Details indicated as below: Figure 2a is the set standard light sources of CIE in the CCT range from $2700 \mathrm{~K}$ to $6500 \mathrm{~K}$ (as CIE A, F4, F3, F11, F2, D50, D55, and D65) [44,45]. Figure 2b is the set standard light sources of CIEF in the 
CCT range from $2700 \mathrm{~K}$ to $6500 \mathrm{~K}$ (as F4, F12, F3, F11, F2, F8, F10, and F7) [44,45]. Figure 2c is the light sources of RGB LEDs in the CCT range from $2700 \mathrm{~K}$ to $12,500 \mathrm{~K}$ [46,47]. Figure $2 \mathrm{~d}$ is the light sources of OLEDs in the CCT range from $2700 \mathrm{~K}$ to $6500 \mathrm{~K}$ [11].
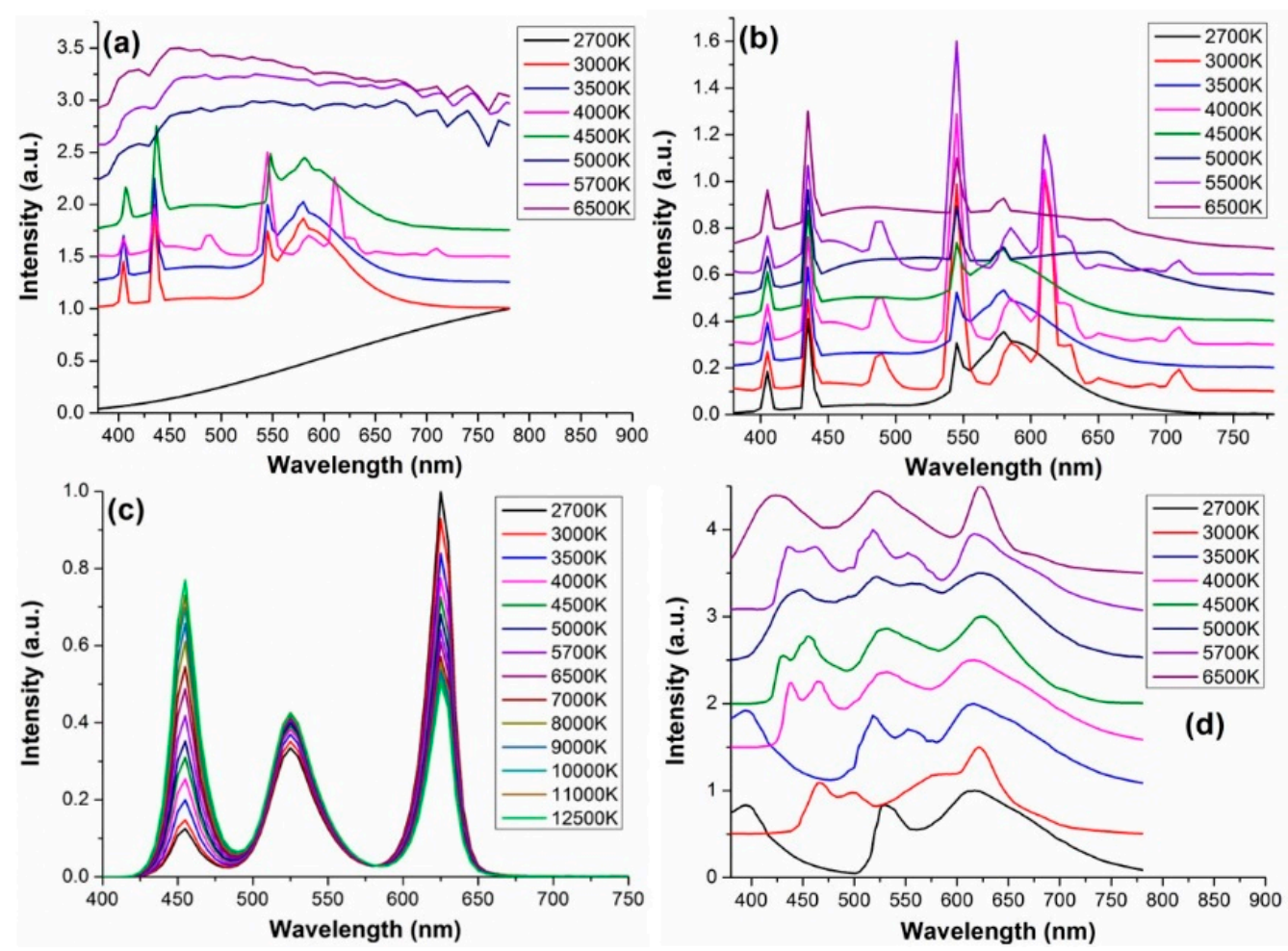

Figure 2. Light sources spectra with different CCT: (a) CIE; (b) CIEF; (c) RGB LED; (d) OLED.

The image can then be reproduced under different light sources and luminosities, and conduct further analysis. A previous study already proved that the reproduced image bears high similarity with the real image. Thus, the simulated image was treated as the real image and simulation will not be mentioned for the rest of this paper.

\section{Results and Analysis}

The CIE DE2000 average color difference was chosen as the basis of this study's analyzing. When two images are located close to each other in the color space, their average color difference is also closer. When two different images are observed, the closer they are located in the color space, the more the two images are found to be similar or even identical. Thus, images with similar or same visual experiences can be categorized by a calculating average color difference. This study compared images under the same set of standard light sources (CIE, CIEF, RGB LED, OLED). Under different CCT and luminosities, a continuous change in the average color difference of the image can be observed when compared to the image reference point (D65 400 lux) using the interpolation method. This information is then combined into an interpolation figure, as shown in Figure 3. 
(a) 50

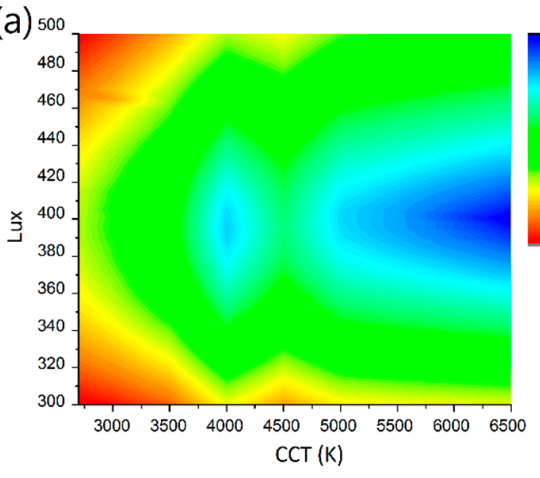

(c)

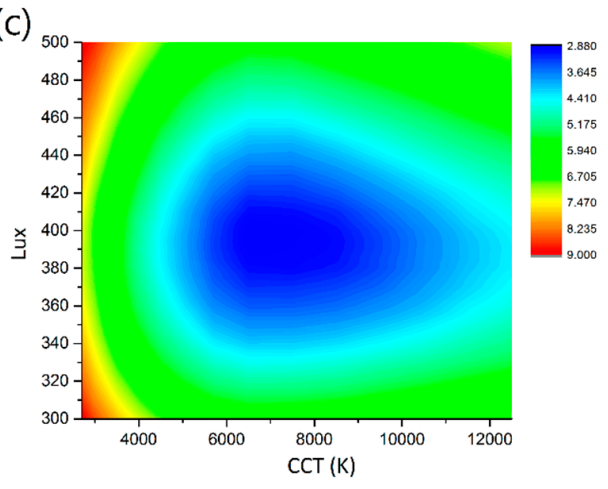

(b)

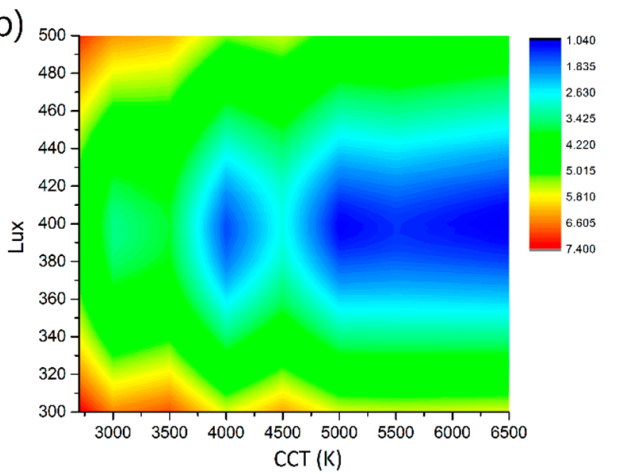

(d)

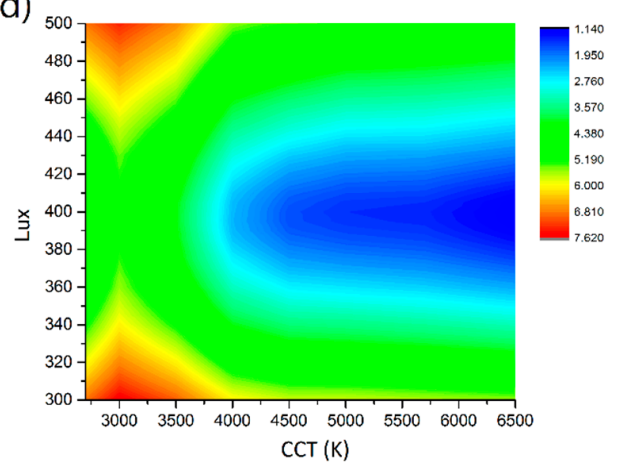

Figure 3. Gradient figures describing the corresponding average color difference under CCT and luminosity under standard reference point (CIE D65 400 lux) with different standard light sources: (a) CIE; (b) CIEF; (c) RGB LED; (d) OLED.

\subsection{CIE Light Source}

The interpolation figure of the CIE light source is shown in Figure 3a. The CIE light source exhibited a tendency for increased average color different when the point is farther from the image reference point. A comparison of the points with the same average color difference showed a curve line located that resulted from an increase in the absolute value of its slope when the point are closer to the reference point (higher CCT). Thus, to reach a similar visual experience, requires increasing the luminosity to compensate for the loss of CCT in the lower color temperature region.

However, a closer look at the CCT regions $5000 \mathrm{~K}$ to $4000 \mathrm{~K}$ shows a discontinuous relationship in terms of the reduction of the color difference. The same luminosity can also be observed because increasing the CCT will still result in the same visual experience (same color difference). This result differs from common results obtained in previous studies, where higher luminosity is required to compensate for the reduction of CCT [18]. The discontinuous behavior could be the result of the choice of standard light source: that is, the use of D-series light source from $6500 \mathrm{~K}$ to $5000 \mathrm{~K}$ but F-series from $4500 \mathrm{~K}$ to $2700 \mathrm{~K}$. The lack of data prevents the discussion of the two regions separately, and thus, the CIE light source will not be analyzed.

\subsection{CIE F Light Source}

The interpolation figure of the CIEF light source is shown in Figure $3 \mathrm{~b}$. The figure shows that this light source displayed a more discontinuous behavior compared to the CIE light source. In general, the curve displayed a tendency similar to the CIE light source. However, the CCTs do not have a significant effect on the visual experience at around $5000 \mathrm{~K}$ to $6500 \mathrm{~K}$. This effect could be attributed to the emitting principle of the florescent light source, which resulted in huge spectrum differences. If one looked at the spectral power distribution of CIEF light source (Figure 2b), one can observe that the principal component of the SPD (peaks of the SPD) are different. Because of this discontinuous data set, the CIEF light source is also removed from the scope of this paper's discussion. 


\subsection{RGB LED}

RGB LED displays smooth and symmetrical curves in its interpolation figure, as shown in Figure 3c. This relatively smoother and symmetrical behavior is caused by the emitting principle of RGB LEDs. That is, RGB LED lights are created by mixing three primary colors as one can see from the light source spectrum in Figure 2c. Thus, the interpolation method can efficiently approximate CCTs in between different light sources. This emitting principle also eliminates discontinuity between light sources, resulting in a decent data set for us to study. At first glance, we can determine the points located further away from the center points, which have a larger color difference. Under the same color difference, the lower the CCT is, in order to reach the same visual experience, the larger the change in luminosity is required to compensate for the change in CCT. This difference has the same relationship with CIE and CIEF light sources. The center point locates in the center of the interpolation figure, and thus, the following observation can be made. The figure displayed symmetrical behavior between top and bottom. Hence, in order to reach the same visual experience, changing the same amount of luminosity from the center point implies changing the same amount of CCT. Based on this behavior, this paper will only discuss the part with lower luminosity in the interpolation figure. The relationship of the upper part can be simply obtained through mathematic transformation, which is discussed in Section 5. Different CCTs will have different visual experiences under the same luminosity. Thus, the points on the left and right side of the center point with the same color difference may actually have different visual experiences. The fact that they present the same color difference can be attributed to the calculation of the average color difference. However, a better way to measure visual experience quantitatively does not exist. Hence, the data had to be separated manually into higher and lower CCT regions based on center point and discussed separately.

\subsection{OLED}

The interpolation figure of OLED, as shown in Figure 3d, strongly resembles that of RGB LED. The main difference is that the center point is located on the highest possible CCT (6500 K), which makes the curves smoother. We also observe points of discontinuity at CCT $2700 \mathrm{~K}$, which could likely be attributed to the switching from $\mathrm{P}$ series OLED to $\mathrm{H}$ series. The change in light source type affecting the spectrum implied that interpolation might not be appropriate. Hence, all data at $2700 \mathrm{~K}$ were removed to avoid this error. Overall the interpolation figure displayed a symmetric behavior between the top and bottom parts. Points locate further away the center points had larger color difference. Under the same color difference and in high CCT region, a smaller change in luminosity is required to compensate for loss of CCT. Analysis of the lower luminosity part of the figure is similar to what has been discussed in previous section.

\subsection{Analysis on Different Reference Points}

How do the different reference points affect the results? A good and representative relation between CCT and luminosity should not include color difference, and thus the influence caused by the choice of arbitrary reference point should be as low as possible. We choose a different image reference point and compare the results. Because of the continuity of RGB LED, the 300 lux $2700 \mathrm{~K}$ in RGB LED was used as reference point. This reference point is located on the other end of the interpolation figure compare to the original center. The result is shown in Figure 4a and is a reconstruction of the interpolation figure. Overall, the figure exhibited a similar trend as that of the previous interpolation figure. Points located further away from the center points have a larger color difference. The smaller the CCT, the larger the change in luminosity required to compensate for the difference in CCT under the same color difference. The figure also shows that the CCT region displays a different behavior than that of the lower CCT region. Such behavior can also be observed in the original interpolation figure. These preliminary behavior results suggest that the change in reference point does not affects 
the relationship between CCT and luminosity. The effects of the different reference points thoroughly are discussed in the next section.
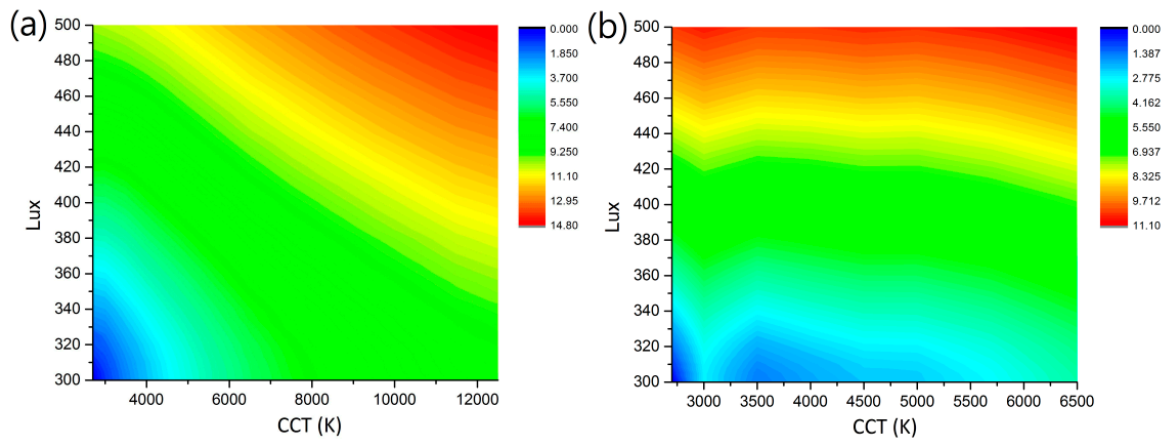

Figure 4. Interpolation figures describing the corresponding average color difference under CCT and luminosity with different standard light sources under a reference point at 300 lux $2700 \mathrm{~K}$ with different standard light sources: (a) RGB LED; (b) OLED.

\subsection{Relationship Analysis}

This study used the interpolate method to respectively plot points with equal color difference and discuss the relationship between CCT and luminosity further. These are referred to as the equal color difference curves. This study conducted regression to determine the best way to describe these curves. From the likely regression lines it was determined that exponential fitting can best describe the relationship between luminosity under the same color difference. The results are shown in Figure 5 and Table 1. Table 1 show all regression results and fitting numbers. The table shows that the RGB LED regression lines have extremely high correlation. All R-squares are about 0.99 , implying that our regression line can describe the relationship between CCT and luminosity almost perfectly. This result may be because of the emitting principle of RGB LED. The fact that the entire light source spectrum is a mix of red, green, and blue lights makes the change on color temperature continuous. OLED regression result indicated a very high correlation. The R-squares also reached around 0.9. Although these results are not as perfect as that obtained for the RGB LED, such result is expected because of the difference in emitting principle. This difference can also be observed from the change on R-squares. As the color difference increases, the R-square gradually decreases. The exponential regression lines can very well describe the relationship between CCT and luminosity. Hence, this regression line can provide us with information as to how the same visual can be reached with different CCT and luminosity. Other fitting constants are discussed in the next section.

Table 1. Regression results and fitting variables for the equal color difference curves.

\begin{tabular}{cccccc}
\hline \multicolumn{3}{c}{ Exponential Fitting } & \multicolumn{3}{c}{ Form: $\mathbf{y}=\mathbf{y}_{\mathbf{0}}+\mathbf{A e}^{\mathbf{r}_{\mathbf{0}} \mathbf{x}}$} \\
\hline Color Difference & $\mathbf{y}_{\mathbf{0}}$ & $\mathbf{A}$ & $\mathbf{r}_{\mathbf{0}}$ & $\mathbf{c h i}$ & $\boldsymbol{R}^{\mathbf{2}}$ \\
\hline OLED & & & & & \\
\hline 2.4 & 359.3125 & $36,996.66$ & -0.0018 & 9.74213 & 0.92705 \\
2.7 & 352.4438 & $18,190.29$ & -0.00167 & 6.16192 & 0.92993 \\
3 & 345.7124 & 6373.249 & -0.00145 & 4.80766 & 0.92172 \\
3.3 & 339.712 & 4112.206 & -0.00137 & 4.65922 & 0.90131 \\
3.6 & 333.4818 & 1983.748 & -0.00121 & 2.63293 & 0.92772 \\
3.9 & 328.3302 & 2704.68 & -0.00132 & 2.69962 & 0.91125 \\
4.2 & 322.6771 & 1813.023 & -0.00124 & 2.18214 & 0.91401 \\
4.5 & 317.4525 & 1684.778 & -0.00124 & 2.22738 & 0.89761 \\
4.8 & 312.0153 & 1156.717 & -0.00117 & 1.59685 & 0.90941 \\
5.1 & 307.0783 & 1325.591 & -0.00123 & 1.43558 & 0.90484 \\
5.4 & 302.1877 & 1278.015 & -0.00124 & 1.37708 & 0.8913 \\
\hline
\end{tabular}


Table 1. Cont.

\begin{tabular}{cccccc}
\hline \multicolumn{3}{c}{ Exponential Fitting } & \multicolumn{3}{c}{ Form: $\mathbf{y}=\mathbf{y}_{\mathbf{0}}+\mathbf{A e}^{\mathbf{r}_{\mathbf{0}} \mathbf{x}}$} \\
\hline Color Difference & $\mathbf{y}_{\mathbf{0}}$ & $\mathbf{A}$ & $\mathbf{r}_{\mathbf{0}}$ & $\mathbf{c h i}$ & $\mathbf{R}^{\mathbf{2}}$ \\
\hline RGB LED & & & $6500 \mathrm{~K}-12,500 \mathrm{~K}$ & \\
\hline 3.8 & 348.4514 & 0.00169 & $9.18 \times 10^{4}$ & 0.17738 & 0.99838 \\
4.1 & 339.7523 & 0.00738 & $7.45 \times 10^{4}$ & 0.71652 & 0.99663 \\
4.4 & 330.6664 & 0.07979 & $5.08 \times 10^{4}$ & 0.31164 & 0.99877 \\
4.7 & 321.7622 & 0.35676 & $3.67 \times 10^{4}$ & 0.37569 & 0.99702 \\
5 & 314.8159 & 0.51721 & $3.25 \times 10^{4}$ & 0.32119 & 0.99628 \\
5.3 & 307.0196 & 1.04373 & $2.62 \times 10^{4}$ & 0.63982 & 0.98967 \\
5.6 & 300.7108 & 1.256 & $2.42 \times 10^{4}$ & 0.45611 & 0.99101 \\
5.9 & 293.7972 & 1.81156 & $2.09 \times 10^{4}$ & 0.63311 & 0.98469 \\
\hline RGB LED & & & $4000 \mathrm{~K}-6500 \mathrm{~K}$ & & \\
4.4 & 332.481 & $338,411.1$ & -0.00191 & 0.34216 & 0.99955 \\
4.7 & 322.6118 & 9543.561 & -0.00122 & 0.26837 & 0.99898 \\
5 & 315.68 & 4580.892 & -0.00109 & 0.43825 & 0.99748 \\
5.3 & 310.5442 & 4810.612 & -0.00114 & 0.32832 & 0.99911 \\
5.6 & 304.1548 & 2904.569 & -0.00105 & 0.15723 & 0.9994 \\
5.9 & 299.9801 & 4196.213 & -0.00117 & 0.82672 & 0.99871 \\
6.2 & 293.0498 & 1721.424 & $-9.78 \times 10^{4}$ & 0.0214 & 0.99994 \\
\hline
\end{tabular}
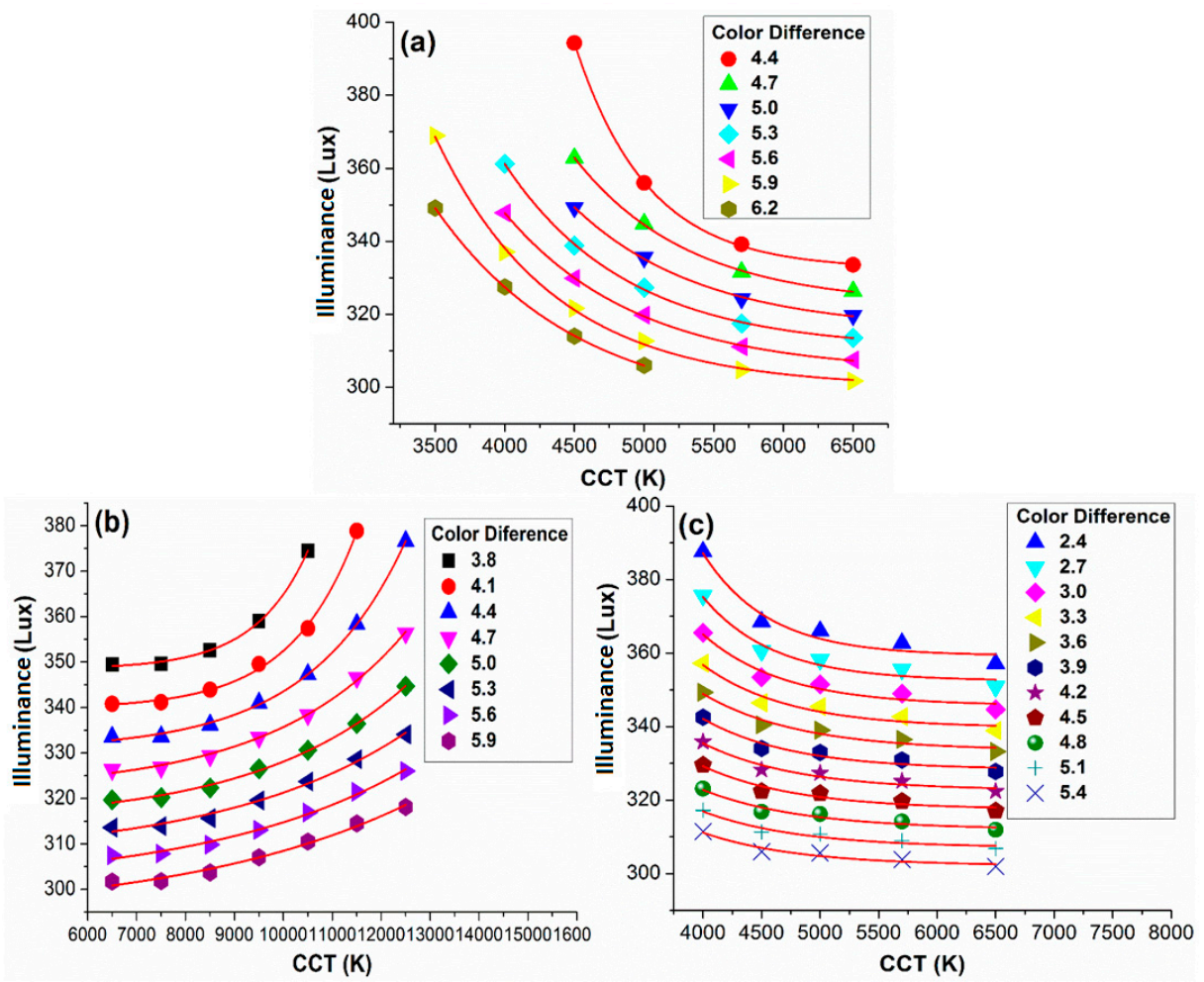

Figure 5. Equal color difference curves under different light source and different temperature region: (a) RGB LED low temperature (3500 K-6500 K); (b) RGB LED high temperature (6500 K-12,500 K); (c) OLED all temperature (4000 K-6500 K). 


\section{Discussion}

Figure 5 shows that using exponential fit on the relationship between CCT and luminosity is appropriate and accurate. This study proposes the following relationship as the fitting function:

$$
\mathrm{y}=\mathrm{y}_{0}+\mathrm{Ae}^{\mathrm{r}_{0} \mathrm{x}}
$$

where $y$ is the corresponding luminosity and $x$ is the CCT. All the fitting variables may vary in term of color difference and light source, as shown in Table 1. This relationship allows for the creation of a transformation when it comes to designing LED lightings. This smart LED transformation allowed this study to achieve metamerism when it comes to lighting design as well as coming up fairer color evaluation metrics removing the influence of CCT and visual experience.

\subsection{Fitting Variables}

Table 1 shows the behaviors of the fitting variables: $r_{0}$ remains constant for the same light source; a decreases as the color difference increases in the OLED and low temperature region of RGB LED, but increases in high temperature region of RGB LED; and $\mathrm{y}_{0}$ decreased slightly as color difference increases. The $\mathrm{r}_{0}$ decides the direction of the exponential curve. However, because the direction of the exponential fitting line in the high temperature region of RGB LED differs from that of the other two, its $r_{0}$ is negative and the change in the variable may be the opposite of that of the others. We will discuss the transformation of the variable further in the section of symmetrical transformation. In this section, the focus is on the low temperature region of the RGB LED and OLD primary. Moreover while some of the changes in the high temperature region of the RGB LED may differ, the physics explanation is exactly the same as that of the others.

Equation (1) can change into the following form simply by moving the variables:

$$
\frac{\mathrm{y}-\mathrm{y}_{0}}{\mathrm{~A}}=\mathrm{e}^{\mathrm{r}_{0} \mathrm{x}}
$$

When the exponential reaches $0, \mathrm{y}_{0}$ is the luminosity at that point. However, CCT reaching either 0 or infinite is very unlikely. Thus, this number do not provide a very meaningful physical implication. However, because what is being discussed is the bottom part of the curve, as the color difference increases, the curve is further away from the center point, thus $\mathrm{y}_{0}$ become smaller. A and $\mathrm{r}_{0}$ represent the weights of $y$ and $x$ respectively. The greater the number $A$ or $r_{0}$, the less effect luminosity has in controlling visual experiences. Under the same change of color difference, a larger change in luminosity is required corresponding to the change in CCT in order to reach the same visual experience. Data show that $r_{0}$ stays fixed while A decreases as color difference increases in OLED and low temperature region of RGB LED, but increases in the high temperature region of RGB LED. Under the same luminosity and the further a point is from the center, A gradually decreases as the color difference increases. This gradually decrease result in a larger change in the CCT required to yield the same visual experience, indicating that an equal color difference curve will have smaller curvature in those regions and vice versa. In both OLED and RGB LED cases, this gradually decrease will also result in a flatter curve around the 5000-6000 K transition region, indicating a similar visual experience in larger range of CCT changes. These findings also correspond to the study by Pedro and his colleagues [18] and effectively provide mathematical proof of their claim that the area with the worst color recognition is that around $5000 \mathrm{~K}$.

\subsection{Symmetrical Transformations}

Figure 6 is a schematic of the equal color difference curve. At most, two lines of symmetry can be obtained (OLED has only one). For convenience, this study assumes that these lines are totally symmetrical at the bottom and at the top as well as the right and left. A set of equal color difference 
curve is first obtained through fitting. Without losing generality, it can be assumed that the following equation can be obtained for the red curve:

$$
\mathrm{y}=\mathrm{y}_{1}+\mathrm{A}_{1} \mathrm{e}^{\mathrm{r}_{1} \mathrm{x}}
$$

The green curve, which is in bilateral symmetry with the red curve in the line of $X=a$, can be obtained using the following equation:

$$
\mathrm{y}=\mathrm{y}_{1}+\mathrm{A}_{1} \mathrm{e}^{\mathrm{r}_{1}(2 \mathrm{~A}-\mathrm{x})}=\mathrm{y}_{1}+\mathrm{A}_{1} \mathrm{e}^{\left(2 \mathrm{Ar}_{1}-\mathrm{r}_{1} \mathrm{x}\right)}=\mathrm{y}_{2}+\mathrm{A}_{2} \mathrm{e}^{\mathrm{r}_{2} \mathrm{x}}
$$

where $\mathrm{A}_{2}=\mathrm{A}_{1} \mathrm{e}^{2 \mathrm{Ar}}$ and $\mathrm{r}_{2}=-\mathrm{r}_{1}$.

Similarly, the purple curve, which is in bilateral symmetry with the red curve in the line of $Y=b$, can be obtained as follows

$$
(2 b-y)=y_{1}+A_{1} e^{r_{1} x}
$$

The equation can be arranged into the following form:

$$
y=\left(2 b-y_{1}\right)-A_{1} e^{r_{1} x}=y_{2}+A_{2} e^{r_{2} x}
$$

where $A_{2}=-A_{1}$ and $y_{2}=2 b-y_{1}$.

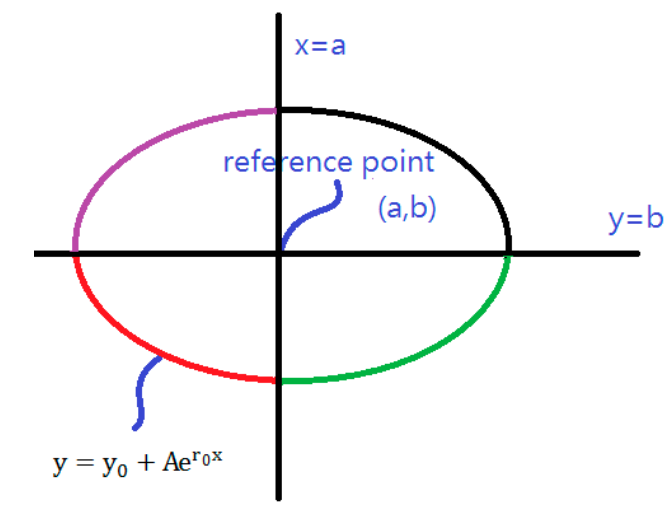

Figure 6. Schematic diagram of equal color difference curve with two sets of perfect symmetric lines.

The availability of our exponential model on other part of the curve can be proven from these relationships. These equations can also be utilized in building relationship models in the future.

\subsection{Switching Reference Point}

Figure $4 \mathrm{a}$,b show two interpolation figures using 300 lux $2700 \mathrm{~K}$ as the image reference point for RGB LED and OLED respectively. A comparison with the original graph (Figure 3c,d), shows an equal color difference curve with almost the same behavior on most regions. This result can be attributed to the change in reference affecting only the color difference instead of the real visual experience. Moreover, color difference is not a part of the equation for relationship between the CCT and luminosity. The main difference lies in the region close to the center point. The region with a different visual experience can have similar color difference when the region is located close to the center. Hence, to prevent misrepresentation of data, this study removes all points within one color difference compared to the center point. In the case of the interpolation figures in Figure 3, the difference is 3.8 for RGB LED and 2.4 for OLED. The application of this rule is recommended in building future models. In Figure 4a, the equal color difference curve at color difference 3.7 and 11 have significantly different trends in its exponential curve. For the fitting variable, line of color difference $3.7 \mathrm{~A}$ is negative and $\mathrm{R}$ is positive, whereas that for color difference is positive for $\mathrm{A}$ and 
negative for R. The behavior of these curves does not follow the same trend when color difference is increased. The same trend can be observed in the case of OLED (Figure $4 \mathrm{~b}$ ), these results indicated that different color difference regions will have different relationship for CCT and luminosity when reaching for the same visual experience. However, this result might require a larger range of CCT light source to generate more data to provide solid proof of this claim.

\section{Conclusion and Future Work}

Using HSI technique combined with color reproduction technique, this smart LED imaging process allowed this study to recreate visual experiences under different standard light sources (CIE, CIEF, RGB LED, OLED), luminosities, and CCT. The results within the same series of standard light sources were compared by analyzing the average color difference. An interpolation figure was constructed to determine how luminosity and CCT affect visual experience and to calculate the equal color difference line to understand its relation. The following conclusions were reached:

(1) The emitting principle causes CIE and CIEF light sources to exhibit a discontinuous behavior in its relation between luminosity and CCT, making it difficult to conduct further analysis on these light sources.

(2) RGB LED and OLED present an exponential relationship on luminosity and CCT for points with similar visual experience. This relationship can be obtained through a simple regression method and utilized further in transforming luminosity and CCT while maintaining the same visual experience.

(3) Mathematical formulas are constructed under symmetrical principle around the center points. These formulas can be used for quick construction of the relationship between CCT and luminosity under different color differences or center points.

(4) The choice of center points (color reference points) does not affect the relationship between luminosity and ССТ. However, we believe that it will affect the valid region of effect.

The relationship between luminosity and CCT presents an option to remove the effects of CCT in color evaluation standards and offers a guideline for adjusting the visual experience solely by adjusting luminosity when creating lighting system. While this relationship is valid only under RGB LED and OLED, the authors of this study believe that future work can be done aiming to create a relationship that can also be valid under other light sources with careful analysis of emitting principle and a well-designed categorization. This relationship may also be integrated into the color evaluation metrics to create a more valid metric for LED light sources. The authors of this study also hope to analyze on other nature colored surface such as human skin and nature material to verify and perfect the created transformation relationship.

Acknowledgments: This research was supported by the Ministry of Science and Technology, the Republic of China, under the Grants MOST 104-2221-E-194-054, 105-2923-E-194-003-MY3, and 105-2112-M-194-005.

Author Contributions: Yu-Sheng Huang and Wei-Cheng Luo were responsible for finishing data and optical calculations; Shih-Wei Feng and Chie-Tong Kuo analyzed color lighting results; Hsiang-Chen Wang and Chia-Mei Lu organized and wrote manuscript.

Conflicts of Interest: The authors declare no conflict of interest.

\section{References}

1. De Almeida, A.; Santos, B.; Paolo, B.; Quiheron, M. Solid state lighting review-Potential and challenges in Europe. Renew. Sustain. Energy Rev. 2014, 34, 30-48. [CrossRef]

2. Humphreys, C.J. Solid-state lighting. MRS Bull. 2008, 33, 459-470. [CrossRef]

3. Azevedo, I.L.; Morgan, M.G.; Morgan, F. The transition to solid-state lighting. Proc. IEEE 2009, 97, 481-510. [CrossRef]

4. Tsao, J.Y.; Saunders, H.D.; Creighton, J.R.; Coltrin, M.E.; Simmons, J.A. Solid-state lighting: An energy-economics perspective. J. Phys. D Appl. Phys. 2010, 43, 354001. [CrossRef] 
5. Sun, C.C.; Moreno, I.; Lo, Y.C.; Chiu, B.C.; Chien, W.T. Collimating lamp with well color mixing of red/green/blue LEDs. Opt. Express 2012, 20, A75-A84. [CrossRef] [PubMed]

6. Crawford, M.H. LEDs for solid-state lighting: Performance challenges and recent advances. IEEE J. Sel. Top. Quantum Electron. 2009, 15, 1028-1040. [CrossRef]

7. Falchi, F.; Cinzano, P.; Elvidge, C.D.; Keith, D.M.; Haim, A. Limiting the impact of light pollution on human health, environment and stellar visibility. J. Environ. Manag. 2011, 92, 2714-2722. [CrossRef] [PubMed]

8. Commission Internationale de l'Éclairage (CIE). Method of Measuring and Specifying Colour Rendering Properties of Light Sources; CIE 13.3; Central Bureau of the CIE: Vienna, Austria, 1995; Volume 20, p. 212.

9. Commission Internationale de l'Éclairage (CIE). Colour Rendering; TC 1-33 Closing Remarks, CIE 135/2; CIE Publication: Vienna, Austria, 1999.

10. Davis, W.; Ohno, Y. Color quality scale. Opt. Eng. 2010, 49, 033602. [CrossRef]

11. Chen, L.Y.; Chen, S.H.; Dai, S.J.; Kuo, C.T.; Wang, H.C. Spectral design and evaluation of OLEDs as light sources. Org. Electron. 2014, 15, 2194-2209. [CrossRef]

12. Yang, X.; Zhou, G.; Wong, W.Y. Recent design tactics for high performance white polymer light-emitting diodes. J. Mater. Chem. C 2014, 2, 1760-1778. [CrossRef]

13. Ying, L.; Ho, C.L.; Wu, H.; Cao, Y.; Wong, W.Y. White Polymer Light-Emitting Devices for Solid-State Lighting: Materials, Devices, and Recent Progress. Adv. Mater. 2014, 26, 2459-2473. [CrossRef] [PubMed]

14. Chen, S.; Tan, G.; Wong, W.Y.; Kwok, H.S. White Organic Light-Emitting Diodes with Evenly Separated Red, Green, and Blue Colors for Efficiency/Color-Rendition Trade-Off Optimization. Adv. Funct. Mater. 2011, 21, 3785-3793. [CrossRef]

15. Komoda, T.; Ide, N.; Varutt, K.; Yamae, K.; Tsuji, H.; Matsuhisa, Y. High-performance white OLEDs with high color-rendering index for next-generation solid-state lighting. J. Soc. Inf. Disp. 2011, 19, 838-846. [CrossRef]

16. Liu, J.; Zhou, Q.G.; Cheng, Y.X.; Geng, Y.H.; Wang, L.X.; Ma, D.G.; Jing, X.B.; Wang, F.S. The first single polymer with simultaneous blue, green, and red emission for white electroluminescence. Adv. Mater. 2005, 17, 2974-2978. [CrossRef]

17. Ohno, Y. Spectral design considerations for white LED color rendering. Opt. Eng. 2005, 44, 111302. [CrossRef]

18. Pardo, P.J.; Suero, M.I.; Pérez, Á.L.; Martínez-Borreguero, G. Optimization of the correlated color temperature of a light source for a better color discrimination. J. Opt. Soc. Am. A 2014, 31, A121-A124. [CrossRef] [PubMed]

19. Thornton, W.A. Color-discrimination index. J. Opt. Soc. Am. 1972, 62, 191-194. [CrossRef] [PubMed]

20. Thornton, W.A. A validation of the color-preference index. J. Illum. Eng. Soc. 1974, 4, 48-52. [CrossRef]

21. Pardo, P.J.; Cordero, E.M.; Suero, M.I.; Pérez, Á.L. Influence of the correlated color temperature of a light source on the color discrimination capacity of the observer. J. Opt. Soc. Am. A 2012, 29, A209-A215. [CrossRef] [PubMed]

22. Luo, M.R.; Cui, G.; Rigg, B. The development of the CIE 2000 colour-difference formula: CIEDE2000. Color Res. Appl. 2001, 26, 340-350. [CrossRef]

23. Commission Internationale de l'Éclairage (CIE). A Color Appearance Model for Color Management Systems: CIECAM02; CIE 159; CIE Publication: Vienna, Austria, 2004; Volume 31, p. 158.

24. Luo, M.R.; Cui, G.; Li, C. Uniform colour spaces based on CIECAM02 colour appearance model. Color Res. Appl. 2006, 31, 320-330. [CrossRef]

25. Hashimoto, K.; Yano, T.; Shimizu, M.; Nayatani, Y. New method for specifying color-rendering properties of light sources based on feeling of contrast. Color Res. Appl. 2007, 32, 361-371. [CrossRef]

26. Kruithof, A.A. Tubular luminescence lamps for general illumination. Philips Tech. Rev. 1941, 6, 65-96.

27. McNelis, J.F.; Howley, J.G.; Dore, G.E.; DeLancy, W.B. Subjective appraisal of colored scenes under various fluorescent lamp colors. Light. Des. Appl. 1985, 15, 25-29.

28. Fotios, S.; Levermore, G.J. Perception of electric light sources of different colour properties. Light. Res. Technol. 1997, 29, 161-171. [CrossRef]

29. Ju, J.; Chen, D.; Lin, Y. Effects of correlated color temperature on spatial brightness perception. Color Res. Appl. 2012, 37, 450-454. [CrossRef]

30. Houser, K.W. Lighting for quality. Light. Des. Appl. 2002, 32, 4-7.

31. Guo, X.; Houser, K.W. A review of colour rendering indices and their application to commercial light sources. Light. Res. Technol. 2004, 36, 183-197. [CrossRef] 
32. Wei, M.; Houser, K.W. Status of solid-state lighting based on entries to the 2010 US DOE Next Generation Luminaire competition. Leukos 2012, 8, 237-259.

33. Houser, K.W.; Wei, M.; David, A.; Krames, M.R.; Shen, X.S. Review of measures for light-source color rendition and considerations for a two-measure system for characterizing color rendition. Opt. Express 2013, 21, 10393-10411. [CrossRef] [PubMed]

34. Boyce, P.R.; Cuttle, C. Effect of correlated colour temperature on the perception of interiors and colour discrimination performance. Light. Res. Technol. 1990, 22, 19-36. [CrossRef]

35. Hu, X.; Houser, K.W.; Tiller, D.K. Higher color temperature lamps may not appear brighter. Leukos 2006, 3, 69-81.

36. Wang, H.C.; Chen, Y.T.; Lin, J.T.; Chiang, C.P.; Cheng, F.H. Enhanced visualization of oral cavity for early inflamed tissue detection. Opt. Express 2010, 18, 11800-11809. [CrossRef] [PubMed]

37. Wang, H.C.; Chen, Y.T. Optimal lighting of RGB LEDs for oral cavity detection. Opt. Express 2012, 20, 10186-10199. [CrossRef] [PubMed]

38. Wang, H.C.; Tsai, M.T.; Chiang, C.P. Visual perception enhancement for detection of cancerous oral tissue by multi-spectral imaging. J. Opt. 2013, 15, 055301. [CrossRef]

39. Hsiao, Y.P.; Wang, H.C.; Chen, S.H.; Tsai, C.H.; Yang, J.H. Identified early stage mycosis fungoides from psoriasis and atopic dermatitis using non-invasive color contrast enhancement by LEDs lighting. Opt. Quantum Electron. 2015, 47, 1599-1611. [CrossRef]

40. Hsiao, Y.P.; Wang, H.C.; Chen, S.H.; Tsai, C.H.; Yang, J.H. Optical perception for detection of cutaneous T-cell lymphoma by multi-spectral imaging. J. Opt. 2014, 16, 125301. [CrossRef]

41. Jen, C.P.; Huang, C.T.; Chen, Y.S.; Kuo, C.T.; Wang, H.C. Diagnosis of human bladder cancer cells at different stages using multispectral imaging microscopy. IEEE J. Sel. Top. Quantum Electron. 2014, 20, 81-88.

42. Wang, H.C.; Huang, S.W.; Yang, J.M.; Wu, G.H.; Hsieh, Y.P.; Feng, S.W.; Lee, M.K.; Kuo, C.T. Large-area few-layered graphene film determination by multispectral imaging microscopy. Nanoscale 2015, 7, 9033-9039. [CrossRef] [PubMed]

43. Wu, I.-C.; Weng, Y.-H.; Lu, M.-Y.; Jen, C.-P.; Fedorov, V.E.; Chen, W.C.; Wu, M.T.; Kuo, C.-T.; Wang, H.C. Nano-Structure $\mathrm{ZnO} / \mathrm{Cu}_{2} \mathrm{O}$ Photoelectrochemical and Self-powered Biosensor for Esophageal Cancer Cell Detection. Opt. Express 2017, 25, 7689-7706. [CrossRef] [PubMed]

44. Lam, Y.M.; John, H.X. Evaluation of the quality of different D65 simulators for visual assessment. Color Res. Appl. 2002, 27, 243-251. [CrossRef]

45. Smith, T.; Guild, J. The C.I.E. colorimetric standards and their use. Trans. Opt. Soc. 1931, 33, 73. [CrossRef]

46. McCamy, C.S. Correlated color temperature as an explicit function of chromaticity coordinates. Color Res. Appl. 1992, 17, 142-144. [CrossRef]

47. Žukauskas, A.; Vaicekauskas, R.; Shur, M. Solid-state lamps with optimized color saturation ability. Opt. Express 2010, 18, 2287-2295. [CrossRef] [PubMed]

(C) 2017 by the authors. Licensee MDPI, Basel, Switzerland. This article is an open access article distributed under the terms and conditions of the Creative Commons Attribution (CC BY) license (http://creativecommons.org/licenses/by/4.0/). 